\title{
Long Term Ophthalmic Follow up in a Case with LCHAD Deficiency
}

\author{
Levy N*, Paz T, Leiba H, Hadas B and Parness R \\ Ophthalmology Department, Kaplan Medical Center, Israel
}

*Corresponding author: Levy Niv, Department of Ophthalmology, Kaplan Medical Center, 1 Pasternak Road, Rehovot 76100, Israel

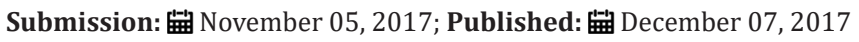

\begin{abstract}
Long-chain 3-hydroxyacyl-CoA dehydrogenase (LCHAD) deficiency is an autosomal recessive disorder of mitochondrial fatty acid beta oxidation, associated with hypoketotic hypoglycemia, hepatic steatosis, rhabdomyolysis, cardiomyopathy and retinal changes. We present the course of retinal findings in a case of a 6-year-old girl with LCHAD deficiency diagnosed at infancy, and hence early treated and followed. Our patient had annualeye exams from the age of 1 year. Clinical examinations, ocular coherence tomography (OCT) and electroretinogram (ERG) findings during follow up are presented. At the age of 3 years, after systemic deteriorations, nyctalopia appeared with pigmentary retinopathy changes in both eyes. ERG was subnormal, while Infra-red reflectance imaging with OCT displayed more advanced stage of the disease. Progressive chorioretinopathy with visual impairment was observed along the follow up on clinical exams, as well as on repeated OCTs and ERGs. In conclusion, retinal dystrophy in children can be related to metabolic disorders, including LCHAD deficiency. The retinal findings may rapidly progress despite dietary treatment, in relation to frequent metabolic decompensations. On early phase of the disease, Infra-red reflectance imaging with OCT may better identify the severity of the chorioretinopathy. Regular follow-up, including OCT, is recommended in LCHAD deficiency patients to monitor the ocular status.
\end{abstract}

Keywords: Long-chain 3-hydroxyacyl-CoA dehydrogenase (LCHAD); Ocular coherence tomography (OCT); Electroretinogram (ERG); Retinal dystrophy

\section{Introduction}

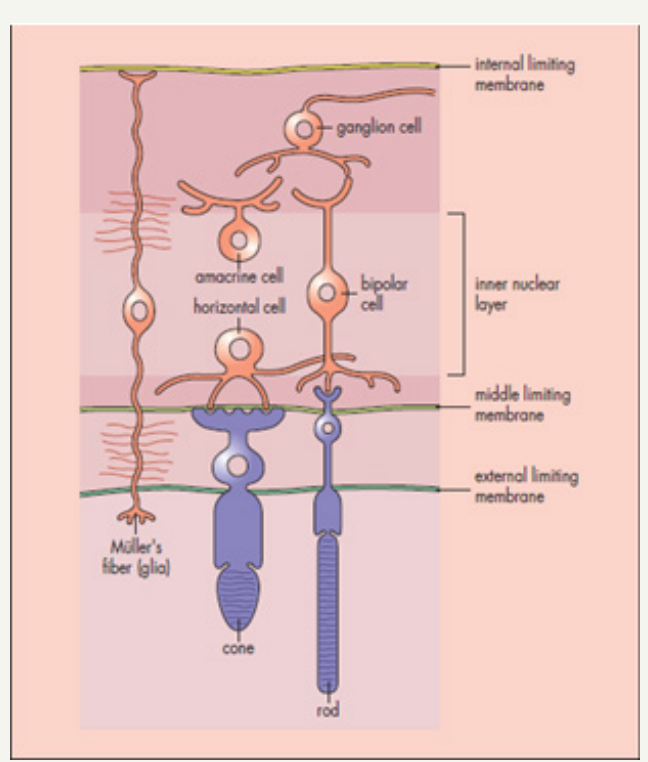

Figure 1: Neuronal connections in the retina and participating cells [5]. The inner nuclear layer contains the nuclei of the bipolar cells (second neuron) and Mullerian glia. The amacrine cells are found on the inside and the horizontal cells on the outside of this layer, next to their respective plexiform connections.
Mitochondrial beta oxidation is the principal pathway by which energy is obtained from fatty acids [1]. Defect in this pathway are associated with hypoketotic hypoglycemia, hepatic steatosis, rhabdomyolysis, cardiomyopathy, polyneuropathy and abnormal urinary metabolites [1-4]. Long-chain 3-hydroxyacylCoA dehydrogenase (LCHAD) deficiency disrupts this pathway and was first described in 1989 [1]. LCHAD is an autosomal recessive disorder [2] with unknown occurrence frequency in Israel or the United States, but in the Swedish population the incidence is $1: 100,000$ [3]. Analysis of the frequency of the most common mutation (G1528C), comprising more than $90 \%$ of mutated alleles in Swedish population [3], revealed a carrier frequency of 1:240 in Finland. The ocular findings associated with this rare metabolic disorder may include pigmentary retinopathy, developmental cataract, progressive myopia, visual fields defects and color vision abnormalities [1]. The neural retina is organized in layers (Figure 1). Essentially, there is the photoreceptor layer plus the bipolar and ganglion cell layer, which represent the outer first neuron and inner second neuron of the visual pathway [5]. In retinal dystrophy secondary to LCHAD deficiency the outer layers of the retina are mainly affected, including the photoreceptor layer comprising rods and cones, and the retinal pigment epithelium (RPE). The human ocular fundus contains a variety of substances that variably absorb, reflect or scatter infra-red light, hence Infra-red reflectance imaging 
has the potential to highlight sub-retinal features and pathology by penetrating further through the fundus than other modalities [6]. Optical coherence tomography (OCT) is a non-invasive imaging technique based on the principle of optical reflectometry light, which enables precise anatomic examination of ocular structures (Figure 2) [7]. This technique utilizes light waves to create the image. It is similar to an ultrasound, except that the reflected and backscattered light is used to create the image $[5,9]$. OCT is a critical tool in the diagnosis and monitoring of ocular diseases involving the retina $[7,9]$. Electroretinogram $(E R G)$ is a diagnostic test that measures the electrical activity generated by cells in the retina in response to a light stimulus (Figure 3). Reduction in light-induced response on the ERG is useful in documenting deteriorating retinal function [10]. Here, we present the course of retinal findings in a case with LCHAD deficiency. Clinical examination, OCT and ERG findings during follow up are presented.

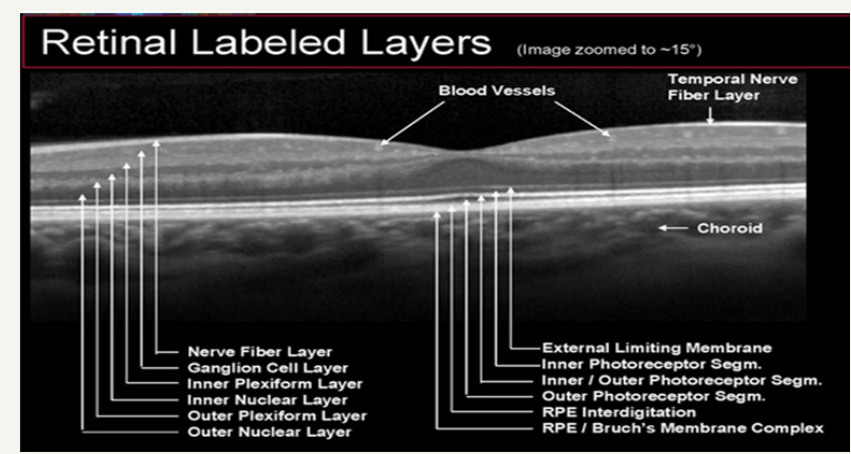

Figure 2: The layers of the retina as demonstrated in a normal Ocular coherence tomography (OCT) [7]. OCT is a non-invasive diagnostic technique that renders an in vivo cross sectional view of the retina by measuring its optical reflections, and is useful in the diagnosis of many retinal conditions $[5,9]$.

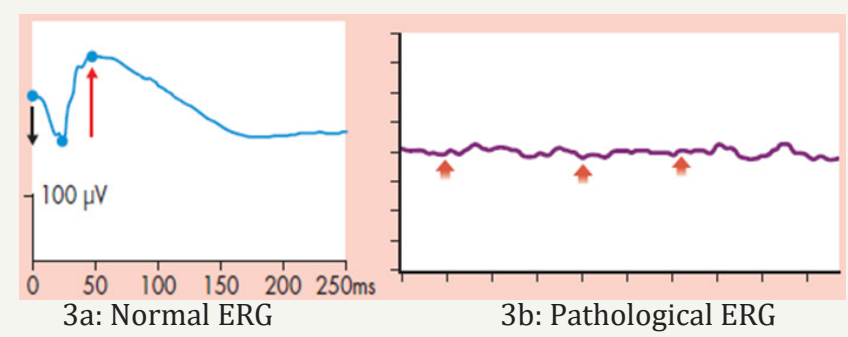

Figure 3: The normal electroretinogram (ERG) records the electrical response evoked from the entire retina by a brief flash of light, and consists of an A-wave (solid black line), which represents the photoreceptor response, and a $B$-wave (solid red line), which represents the combined response of Muller and bipolar cells (Figure 3a). Pathological ERG (Figure $3 \mathrm{~b}$ ) demonstrates markedly diminished rod response to a single flash of bright white light, consistent with the diagnosis of retinal degeneration [5]

\section{Case Presentation}

We present the history of a 6-year-old girl who was diagnosed at infancy with LCHAD deficiency. She was a child of apparently healthy non-consanguineous white parents, born at term after an uneventful pregnancy and a normal delivery. She developed normally until the age of 6 months, when feeding problems and vomiting began.
Subsequently, failure to thrive and motor development stagnation caused her to be hospitalized. Physical examination revealed severe muscle weakness and hypotonia. She cried weakly and was pale and lethargic. The clinical and chemical findings, in connection with the urinary organic acid profile, indicated that she was suffering from a deficiency in fatty acid $\beta$-oxidation, and eventually she was diagnosed as having LCHAD deficiency. Restriction fragment length polymorphism (RFLP) analysis revealed the common G-$->$ C 1528mutation in the LCHAD DNA. She was started on a diet with low-fat high-carbohydrate content and docozahexanoic acid (DHA) supplements, and she had annual eye exams. At the age of 1 year fundoscopy was normal. At the age of 2 years the fovea had a very dark appearance, as a result of perifoveal atrophy. After systemic deterioration and repeated hospitalizations due to hypoglycemia, vomiting and rhabdomyolisis, nyctalopia appeared. Examination at the age of 3 years revealed visual acuity of 6/7.5 in both eyes, and ophthalmoscopy demonstrated a dark macula and a "salt \& pepper" retinopathy extending to the arcades. Infra-red reflectance imaging showed dark center bordered by well-defined hypo-reflective spots, and surrounded by hypo-reflective zone (Figure $4 \mathrm{a} \& 4 \mathrm{~b}$ ). On OCT there were extra-foveal irregularities in the ellipsoid zone of the photoreceptor layer (Figure $4 \mathrm{a} \& 4 \mathrm{~b}$ ). On ERG there were slightly reduced responses. Examination at the age of 6 years revealed deterioration of the visual acuity to 6/30. The patient had myopia in both eye $(-1.5)$, and her color vision was $1 / 13$. Fundoscopy revealed extensive atrophy of the retina and retinal pigment epithelium (RPE) with hypopigmentation and hyperpigmentation in the posterior pole, and pigment clumping in the periphery (Figure $5 b+c$ ). Infra-red reflectance imaging showed a more homogenous hypo-reflectivity of the macula (Figure 6b). OCT showed a decrease in central macular thickness, and atrophy of the photoreceptors layer with flattening of the umbo (Figure 6a). ERG showed severely reduced photopic (cone) and scotopic (rod) responses.

\section{Discussion}

Retinal dystrophy is one of the manifestations of LCHAD deficiency [1-4]. Treatment is aimed at ensuring sufficient caloric intake during periods of metabolic stress and fasting, and DHA supplementation is believed to slow retinal dystrophy [3]. LCHAD activity has been found in human RPE cells, and the metabolic products of the $\beta$-oxidation pathway in this enzyme deficiency might contribute to the retinal damage seen in this rare metabolic disorder [11]. The chorioretinopathy in LCHAD deficiency can be classified into 4 stages [8]: At stage 1, the fundus appears normal to diffusely hypo-pigmented. At stage 2, pigment clumping in the RPE gives rise to pigmentary changes. At stage 3 , the granular fundus pigmentation disappears centrifugally with concomitant atrophy of the choroid and the posterior pole, leaving bare sclera. At stage 4, the central island of the remaining choroids and photoreceptors is lost, and the posterior pole becomes atrophic and devoid of RPE. At the periphery choroidal vessels and RPE remain relatively normal. Late diagnosis with severe symptoms at diagnosis, neonatal hypoglycaemia and frequent decompensations may increase the progression rate of the chorioretinopathy [3]. 


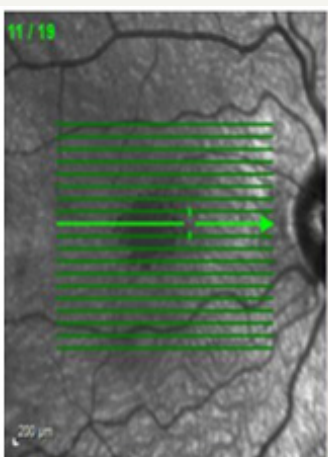

4a: Right Eye

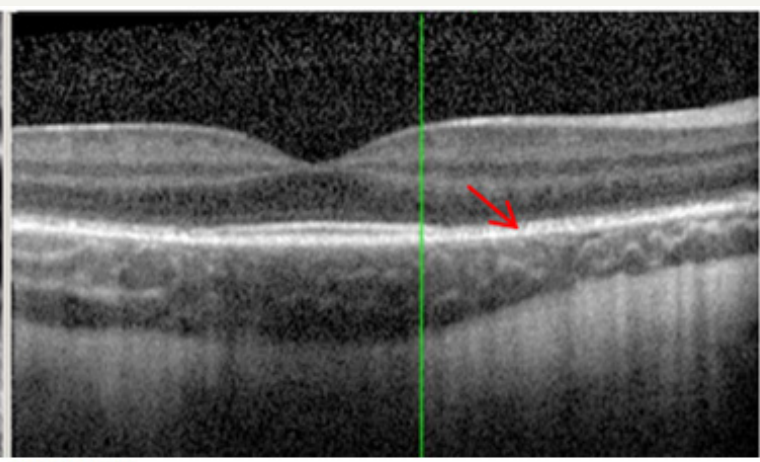

4b: Left Eye

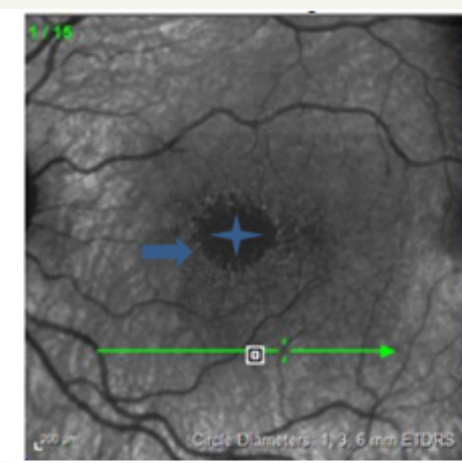

Figure 4: Infra-red reflectance imaging showing a dark center (marked by blue asterisk) bordered by well-defined hypo-reflective spots, and surrounded by hypo-reflective zone (marked by blue arrow) (Figure 4b). OCT showing extra-foveal irregularities in the ellipsoid zone of the photoreceptor layer (marked by red arrow) (Figure 4a).

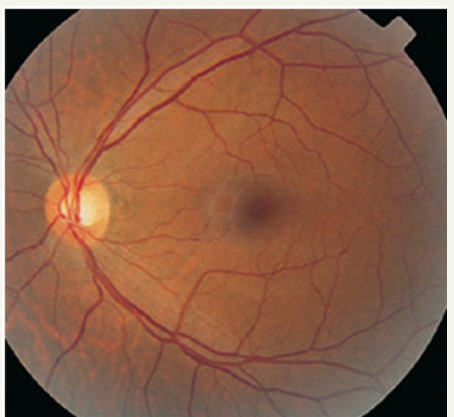

5a: Left Eye; Normal fundus

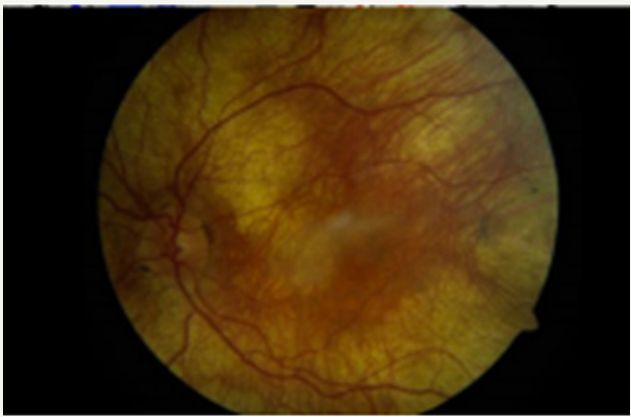

5b: Left Eye

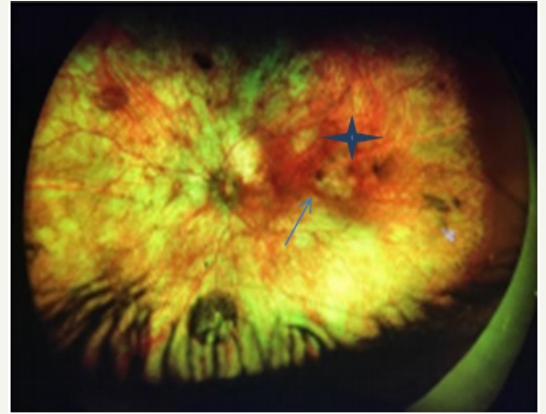

5c: Left Eye; Wide Field

Figure 5: Normal fundus (5a). Fundoscopy revealing extensive atrophy of the retina and RPE with hypopigmentation (blue arrow) and hyperpigmentation (blue asterisk) in the posterior pole, and pigment clumping in the periphery (Figure $5 \mathrm{~b}+\mathrm{c}$ )
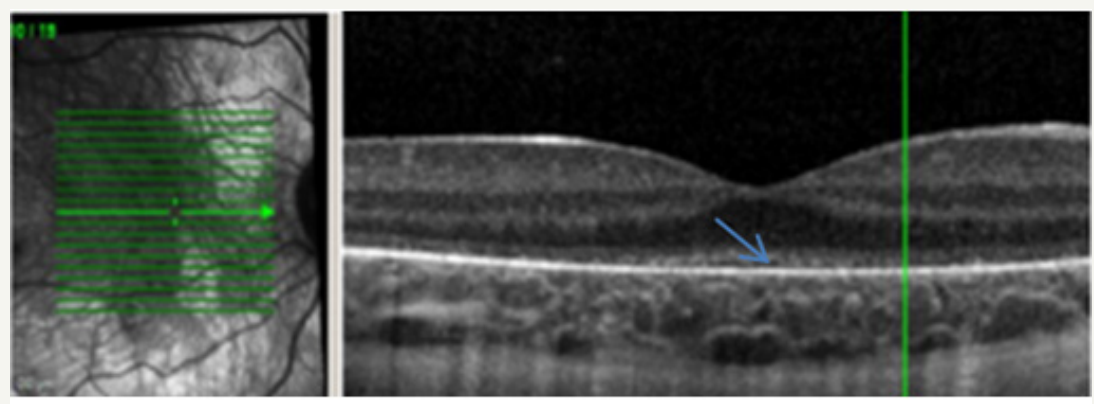

6a: Right Eye

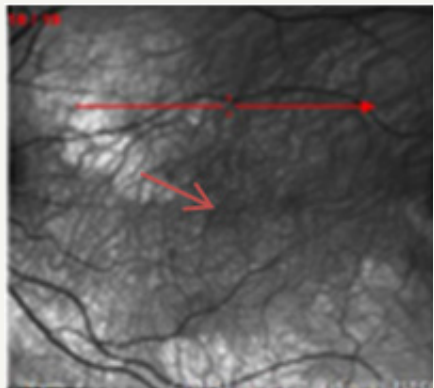

6b: Left Eye

Figure 6: Infra-red reflectance imaging showing a more homogenous hypo-reflectivity of the macula (marked with red arrow) (Figure 6b). OCT showing a decrease in central macular thickness, and atrophy of the photoreceptors layer (marked with blue arrow) with flattening of the umbo (Figure 6a).

This case of a 6-year-old girl with LCHAD deficiency diagnosed at infancy, presented the clinical examination, OCT and ERG findings during follow up, and demonstrated that early diagnosis and adequate therapy may delay but not prevent the progression of retinal dystrophy, as shown in previous case reports [1,3]. LCHAD deficiency is a life-threatening condition and can lead to death due to hepatic or cardiorespiratory illnesses $[4,12,13]$. Progressive pigmentary retinopathy in LCHAD deficiency is a leading cause of blindness in this rare metabolic disorder. Most of the patients with this enzyme deficiency who had ocular manifestations died at a very young age, whereas those who had peripheral neuropathy and chorioretinopathy as the major manifestations survived longer [4]. The early recognition of LCHAD deficiency can increase the life expectancy in these patients through avoiding catabolism and through appropriate diets [2]. Testing for the disorder, therefore, should be included in the diagnostic process for children with 
retinal dystrophy, in particular when other clinical symptoms are known to have occurred, such as history of neonatal hypoglycaemia or failure to thrive $[2,3]$.

Ocular complications beginning with retinal pigmentations and progressing to impaired retinal function with pathological electroretinography (ERG) have been described in case reports and in small case series since the late 1980s.In a case series presenting long-term follow up of ocular complications and ERG findings in ten children with LCHAD deficiency [3], all 10 children developed chorioretinalpathology. Profound chorioretinal atrophy, severe visual impairment and progressive myopia had developed in two teenagers. Milder chorioretinopathy with or without subnormal visual acuity was present in all other children. ERG was pathological in seven children, and demonstrated markedly reduced scotopic and photopic A-wave and B-wave amplitudes. None of the children had an extinguished ERG. The chorioretinopathy was less pronounced in patients with early diagnosis (1st week of life) and early institution of dietary therapy, while it was more severe in children with late diagnosis. Three patients developed myopia, while none had developed cataract at the time of follow-up [3].

ERG and OCT findings have been previously described in a few studies of patients with LCHAD deficiency $[3,4]$. ERG findings showed only subtle changes in implicit times and amplitudes. These do not appear to mirror visual acuity, but may be representative of the gradual but ultimately progressive nature of this condition [1]. Interestingly, progressive myopia has been noted to coincide with the development of staphylomatous like posterior pole changes, and may be a better indicator of disease progression in this dystrophy [1]

New technologies, such as OCT assist in delineating the abnormalities in the retina and RPE [4]. In a previous case report describing two patients with LCHAD deficiency, presenting with visual abnormalities at a later age ( $>10$ years), OCT was performed and showed RPE irregularity and diffuse disruption of the RPE layer in the fovea of both patients [4]

In the case presented here, during the early phase of the disease ERG findings did not reflect the clinical findings. If there is destruction of the retinal receptors, the ERG will decrease and eventually become completely extinguished (Figure 3 ). The implicit time of the b-wave is usually delayed reflecting dysfunction of the retinal neurons before destruction, so that it can be used as an early sign of a progressive disease [2]. A rather good ERG can sometimes occur in secondary retinal degenerations, compared with fundus changes and visual field abnormalities [2]. On the other hand, OCT and infra-red reflectance imaging better demonstrated the severity of the chorioretinopathy. In the early phase of the disease the Infrared reflectance imaging and OCT showed a dark center, bordered by well-defined hypo-reflective spots and surrounded by hyporeflective zone, as a result of the perifoveal atrophy developing early in the disease course. The darker appearance of the macular region may be partly the result of perimacular atrophy, which increases the contrast to the surrounding retina. Thus, OCT and Infra-red reflectance imaging are more sensitive and easier to implement in the early phases of LCHAD deficiency.

\section{Conclusion}

Retinal dystrophy in children can be related to metabolic disorders, including LCHAD deficiency. The retinal findings may rapidly progress despite dietary treatment, in relation to frequent metabolic decompensations. On early phases of the disease, Infrared reflectance imaging and OCT may better identify the severity of the chorioretinopathy. Regular follow-up, including OCT, is recommended in LCHAD deficiency patients to monitor the ocular and systemic status.

\section{References}

1. McGimpsey SJ, Williams M, Mulholland DA (2006) Ten year follow up of pigmentary retinopathy associated with LCHAD deficiency. EYE (20): 1074-1075.

2. Schrijver-weiling I, van Rens GH, Wittebol-Post D, Smeitink JA, de Jaker JP, et al. (1998) Retinal dystrophy in LCHAD deficiency. BJ of Opht 81(4): 291-294.

3. Fahnehjelm KT, Holmstrom G, Ying L, Haglind CB, Nordenstrom A, et al. (2008) Ocular characteristic in 10 children with LCHAD deficiency: a cross-sectional study with long-term follow up. Acta Ophthamol 86(3): 329-337.

4. Turaka K, Bryan JS, Gordon AJ, Kwong HM, Reddy R, et al. (2012) Clinical and image-guided chorioretinal findings in LCHAD deficiency. J Pediarc Endocrinol Metabol 25(5-6): 565-567.

5. Myron Yanoff, Jay S Duker (2014) Ophthalmology. Saunders Elsevier Inc, ( $4^{\text {th }}$ edn) Philadelphia, United States, pp. 419-459.

6. LY A, Nivison-Smith L, Assaad N, Kalloniatis M (2016) Infrared reflectance imaging in age-related macular degeneration. Ophthalmic Physiol Opt 36(3): 303-316.

7. Staurenghi G, Sadda S, Chakravarthy U, Spaide RF (2014) Proposed lexicon for anatomic landmarks in normal posterior segment spectraldomain optical coherence tomography: the IN•OCT consensus. Ophthalmology 121(8): 1572-1578.

8. Tyni T, Kivela T, LappiM, Summanen P, Nikoskelainen E, et al. (1998) Ophthalmological findings in long-chain 3-hydroxyacyl CoA dehydrogenase deficiency caused by the G1528C mutation: a new type of hereditary metabolic chorioretinopathy. Opthalmology 105(5): 810824.

9. Huang D, Swanson EA, Lin CP, Schuman JS, Stinson WG, et al. (1991) Optical coherence tomography. Science 254(5035): 1178-1181.

10. Young B, Eggenberger E, Kaufman D (2012) Current electrophysiology in ophthalmology: a review. Current Opin Ophthalmol 23(6): 497-505.

11. Tyni T, Johnson M, Eaton S, Pourfarzam M, Andrews R, et al. (2002) Mitochondrial fatty acid $\beta$-oxidation in the retinal pigment epithelium. Pediatr Res 52(4): 595-600

12. Wanders RJ, Duran M, Ijlst L, de Jager JP, van Gennip AH, et al. (1989) Sudden infant death and long-chain 3-hydroxyacyl-CoA dehydrogenase. Lancet 2(8653): 52-53.

13. Bertini E, Dionisi-Vici C, Garavaglia B, Burlina AB, Sabatelli M, et al. (1992) Peripheral sensory-motor polyneuropathy, pigmentary retinopathy, and fatal cardiomyopathy in long-chain 3-hydroxy-acyl-CoA dehydrogenase deficiency. Eur J Pediatr 151(2): 121-126. 